\title{
A novel differential diagnostic model for multiple primary lung cancer: Differentially-expressed gene analysis of multiple primary lung cancer and intrapulmonary metastasis
}

\author{
DALI CHEN ${ }^{1}$, LONGYONG MEI ${ }^{2}$, YUBIN ZHOU ${ }^{1}$, CHENG SHEN $^{1}$, \\ HUAN XU ${ }^{3}$, ZHONGXI NIU ${ }^{1}$ and GUOWEI CHE ${ }^{1}$ \\ ${ }^{1}$ Department of Thoracic Surgery, West China Hospital, Sichuan University, Chengdu, Sichuan; ${ }^{2}$ Department of Thoracic \\ Surgery, Daping Hospital, The Third Military Medical University, Chongqing; ${ }^{3}$ Department of Pathology, \\ West China Hospital, Sichuan University, Chengdu, Sichuan, P.R. China
}

Received March 24, 2014; Accepted December 10, 2014

DOI: $10.3892 / \mathrm{ol} .2015 .2880$

\begin{abstract}
The incidence of synchronous multiple primary lung cancer (MPLC) is increasing. However, present diagnostic methods are unable to satisfy the individualized treatment requirements of patients with MPLC. The present study aimed to establish a quantitative mathematical model and analyze its diagnostic value for distinguishing between MPLC and cases of the histologically similar disease, intrapulmonary metastasis (IPM). The sum value of the differential expression ratios of four proteins, namely p53, p16, p27 and c-erbB2, was evaluated by immunohistochemically-staining specimens of primary cancers, second separate cancers, metastatic lymph nodes and metastatic cancers. The sum value of the differential expression ratio of the four proteins from the primary tumor and the lymph-node metastasis or metastatic cancer was $<90$ in the 11 patients with a single metastatic cancer and in the 30 patients with lymph-node metastasis, but was $>90$ in the 14 patients with different histological types of MPLC. Therefore, a quantitative differentially-expressed gene mathematical model was established as follows: Sum of the differential expression ratios $=\mathrm{p} 16 \mathrm{~T} 1-\mathrm{T}+\mathrm{p} 27 \mathrm{~T} 1-\mathrm{T} 2+\mathrm{C}-\mathrm{erbB} 2 \mathrm{~T} 1-\mathrm{T} 2+\mathrm{p} 53 \mathrm{~T} 1-\mathrm{T} 2$, where $\mathrm{T} 1$ is the primary cancer and T2 is the lymph node metastasis, metastatic cancer or the second separate cancer. The quantitative differentially-expressed gene mathematical model is considered to be a useful tool for distinguishing between MPLC and IPM.
\end{abstract}

\section{Introduction}

The rate of morbidity resulting from multiple primary lung cancer (MPLC) is increasing, and in order to devise an

Correspondence to: Professor Guowei Che, Department of Thoracic Surgery, West China Hospital, Sichuan University, 37 Guoxuexiang, Chengdu, Sichuan 610041, P.R. China

E-mail: guowei_che@yahoo.com

Key words: differentially-expressed gene mathematical model, multiple primary lung cancer, intrapulmonary metastasis effective therapeutic strategy, it is important to distinguish between MPLC and intrapulmonary metastasis (IPM). The incidence of synchronous MPLCs in a reported clinical series was between 1 and $7 \%$ (1). Another study reported that up to $10 \%$ of patients who survive a primary lung carcinoma will go on to develop a second primary lung tumor (2). A consensus exists regarding the therapeutic schedule of MPLC, which suggests that surgical treatment confers improved patient prognoses compared with chemotherapy. Therefore, a correct differential diagnosis of MPLC, as opposed to IPM, is conducive to effective individualized treatment. However, based solely on the Martini and Melamed criteria (3), which is widely used in clinical settings, it can be challenging to correctly diagnose MPLC. Furthermore, several cancers, including lung cancers, arise as a result of an accumulation of different genetic and epigenetic alterations (4,5). In a number of organs, carcinogenesis is considered to be a multistep process due to the accumulation of several sequential molecular abnormalities. A previous study identified that the overall frequency of the loss of heterozygosity $(\mathrm{LOH})$ within cell clones progressively increased as the severity of histopathological changes progressed from hyperplasia to dysplasia to carcinoma in situ (6). Furthermore, the incidence of $\mathrm{LOH}$ has been demonstrated to increase along with the histological progression of lung adenocarcinoma (7). The definition of field cancerization has been extended to include cases of multiple primary tumors of the entire upper aerodigestive tract (8). The pathogenesis of multiple primary tumors and metastatic tumors is fundamentally different. Tissues in different fields may develop a unique genetic phenotype under the action of the same carcinogen (e.g., cigarettes) and proceed to form multiple primary tumors. By contrast, metastatic tumors are formed via the hematogenous and/or lymphatic metastasis of primary tumors. Primary and metastatic tumors exhibit a similar origin of clonality. The identification of molecular and genetic variations between tumors will allow for the differential diagnosis of MPLC and IPM. Recent advances in the study of molecular tumorigenesis have demonstrated that the genetic alterations acquired during tumor progression may act as potentially useful markers during clonality analysis. Certain studies have 
suggested that the gene mutational analysis of tumors could be a supplementary method to distinguish between MPLC and IPM (9-11). We have formulated two inclusion criteria in order to identify optimal genetic markers for use during clonality analysis: i) A commonly occurring and independent mutation that occurs in the early stages of disease and is maintained throughout tumor progression; and/or ii) a prognostic marker that is able to evaluate tumor progression. In total, four genetic markers, p53, p16, p27 and c-erbB2, were selected in order to examine the differences in clonality between two separate tumors from the same patient by immunohistochemical (IHC) staining. In addition, the study aimed to establish a quantitative differentially-expressed gene mathematical model to discriminate between cases of MPLC and IPM.

\section{Materials and methods}

Patients and clinical features. Of the 111 consecutive patients with primary lung cancer who had undergone a surgical resection between August 1999 and December 2009 at the Department of Thoracic Surgery, West China Hospital, Sichuan University (Chengdu, China), 50 patients were diagnosed with MPLCs according to the Martini and Melamed criteria (3). Of these patients, 36 exhibited MPLCs of the same histological type, including 34 patients with synchronous MPLCs and two with metachronous MPLCs, while 14 presented with MPLC of a different histological type. Finally, the 36 patients with MPLCs of the same histological type, in which paraffin sections of all tumors were available, were enrolled in the present study. In addition, 20 patients diagnosed with IPM during the same period, according to the Martini and Melamed criteria, were included. In total, 30 patients with lymph node metastasis, 11 with distant metastasis (eight brain metastases, two bone metastases and one adrenal metastasis) and 14 MPLC patients with different histological types were selected as negative or positive controls for the expression analysis of the four proteins between primary tumors and metastases. The clinicopathological data were obtained from a retrospective chart review. The tumor stage was classified according to the 2009 revision of the International System for Staging Lung Cancer (12). The characteristics of the patients with MPLCs, IPM or lymph node metastasis are shown in Table I. The experiments were approved by the West China Hospital Ethics Committee (no. 201333) and all participating patients provided informed consent.

IHC staining. Four proteins, p53, p16, p27 and c-erbB2, which have been demonstrated to be independent prognostic factors for non-small cell lung cancer (NSCLC) (13-16), were selected for the differential diagnostic analysis of MPLC and IPM. IHC staining was performed using serial sections obtained from the same paraffin-embedded blocks. The specimens were stained with hematoxylin and eosin in order to confirm the histological diagnosis. IHC staining was performed using the streptavidin-biotin-peroxidase complex method. For the antigen retrieval, sections were briefly immersed in a citrate buffer ( $0.01 \mathrm{~mol} / 1$ citric acid; $\mathrm{pH}$ 6.0) and then incubated for 25 -min intervals at $100^{\circ} \mathrm{C}$ in a microwave oven. Next, the sections were incubated with a monoclonal mouse anti-p53 antibody (dilution, 1:100; sc-6243, Santa Cruz Biotechnology,
Dallas, TX, USA), a polyclonal rabbit anti-p16 antibody (dilution, 1:200; ab54210, Abcam, Cambridge, MA, USA), a monoclonal mouse anti-p27 antibody (dilution, 1:250; ab32034 Abcam) and a monoclonal mouse anti-c-erbB2 antibody (dilution, 1:100; ab2428, Abcam) overnight in a cold room using a labeled streptavidin biotin kit (Dako LSAB kit; Dako, Carpinteria, CA, USA). The antibodies were diluted in phosphate-buffered saline containing $2 \%$ bovine serum albumin.

Evaluation of the stained specimens. Appropriate positive and negative controls were selected for use in the present study. The slides were independently analyzed by two of the authors who were blinded to the clinicopathological data. A positive result for p53, p16, and p27 expression was defined as the presence of nuclear staining, whereas a positive result for c-erbB2 expression was defined as the appearance of cell membrane staining. Subsequent to the IHC detection of p53, p16, p27 and c-erbB2 in each of the specimens, the percentage of immunoreactive tumor cells in five different randomly-selected fields (magnification, $\mathrm{x} 400$ ) was recorded. The final value for the percentage of positive tumor cells was calculated as the average of the positively-immunostained cells. The extent of immunostaining was scored according to the percentage of positive cells in each tumor specimen as follows: No staining, 0; $1-10 \%$ staining, 10; $11-20 \%$ staining, 20; 21-30\% staining, 30; 31-40\% staining, 40; 41-50\% staining, 50; 51-60\% staining, $60 ; 61-70 \%$ staining, 70; 71-80\% staining, 80; 81-90\% staining, 90; and 91-100\% staining, 100.

\section{Results}

Establishment of the quantitative mathematical model based upon the differentially-expressed gene analysis and its application in the diagnosis of MPLC. First, the differential expression of the four proteins in the the primary tumors and metastatic lesions of 30 patients with lymph node metastasis and in 11 patients with distant metastasis were analyzed and subsequently served as a negative control. The differential expression of p53, p16, p27 and c-erbB2 was compared between the primary lung tumors and the metastatic tumors in the lymph nodes of the 30 patients (Table II). The sum value of the differential expression of the four proteins ranged between 10 and 90 (Fig. 1). Next, the differential expression of the four proteins was compared between the primary lung tumors and the distant metastases. The sum value of the differential expression of the four proteins ranged between 10 and 60 (Figs. 2 and 6). The maximum sum value of the differential expression ratios of the four proteins diagnosed as the same histological type of MPLC was $\leq 90$. By contrast, the sum value of the differential expression ratios of the four proteins ranged between 100 and 220 in the 14 patients diagnosed with MPLCs of different histological types (Figs. 3 and 6). Therefore, it was hypothesized that in the case that the difference between two tumors exceeded a score of 90, the tumors were likely to be different, i.e., MPLCs.

On the basis of the experimental data, a quantitative differentially-expressed gene mathematical model was established as follows: Sum of the differential expression ratios $=$ p16T $1-\mathrm{T} 2+\mathrm{p} 27 \mathrm{~T} 1-\mathrm{T} 2+\mathrm{C}-\mathrm{erbB} 2 \mathrm{~T} 1-\mathrm{T} 2+\mathrm{p} 53 \mathrm{~T} 1-\mathrm{T} 2$, where $\mathrm{T} 1$ is the primary cancer and $\mathrm{T} 2$ is the lymph node 
Table I. Tumor characteristics.

A, Intrapulmonary, distant and lymph node metastases

\begin{tabular}{|c|c|c|c|}
\hline Characteristics & Intrapulmonary metastasis & Distant metastasis & Lymph node metastasis \\
\hline No. of patients & 20 & 11 & 30 \\
\hline Age, years (range) & $62(46-74)$ & $55(42-70)$ & $60(38-72)$ \\
\hline \multicolumn{4}{|l|}{ Gender, n $(\%)$} \\
\hline Male & $12(60)$ & $6(54.5)$ & $22(73.3)$ \\
\hline Female & $8(40)$ & $5(45.5)$ & $8(26.7)$ \\
\hline \multicolumn{4}{|l|}{ Second cancer, n (\%) } \\
\hline Metachronous & $3(15)$ & & \\
\hline Synchronous & $17(85)$ & & \\
\hline \multicolumn{4}{|l|}{ No. of tumors } \\
\hline 2 & 20 & & \\
\hline 3 & & & \\
\hline \multicolumn{4}{|l|}{ Histological type, $\mathrm{n}$} \\
\hline Adenocarcinoma & 15 & 4 & 17 \\
\hline Squamous cell carcinoma & 5 & 4 & 10 \\
\hline Other & & 3 & 3 \\
\hline \multicolumn{4}{|l|}{ p stage $(2009 \text { UICC })^{b}, n$} \\
\hline \multicolumn{4}{|l|}{ IA } \\
\hline \multicolumn{4}{|l|}{ IB } \\
\hline IIA & & & 6 \\
\hline IIB & & & 4 \\
\hline IIIA & 10 & & 18 \\
\hline IIB & 7 & & 1 \\
\hline IV & 3 & 11 & 1 \\
\hline
\end{tabular}

\section{B, MPLC}

\begin{tabular}{|c|c|c|c|}
\hline Characteristics & MPLC total & Same histological type & Different histological type \\
\hline No. of patients & 50 & 36 & 14 \\
\hline Age, years (range) & $61(38-80)$ & & \\
\hline \multicolumn{4}{|l|}{ Gender, n (\%) } \\
\hline Male & $34(68)$ & & \\
\hline Female & $16(32)$ & & \\
\hline \multicolumn{4}{|l|}{ Second cancer, n (\%) } \\
\hline Metachronous & $28(56)$ & & \\
\hline Synchronous & $22(44)$ & & \\
\hline \multicolumn{4}{|l|}{ No. of tumors } \\
\hline 2 & 34 & 13 & \\
\hline 3 & $2^{\mathrm{a}}$ & 1 & \\
\hline \multicolumn{4}{|l|}{ Histological type } \\
\hline Adenocarcinoma & & 33 & 11 \\
\hline Squamous cell carcinoma & & 3 & 11 \\
\hline Other & & & 3 \\
\hline \multicolumn{4}{|l|}{ p stage $(2009 \text { UICC) })^{b}$} \\
\hline IA & & 15 & 2 \\
\hline IB & & 12 & 2 \\
\hline IIA & & 2 & 3 \\
\hline IIB & & 6 & 1 \\
\hline IIIA & & 1 & 2 \\
\hline IIIB & & & 4 \\
\hline IV & & & \\
\hline
\end{tabular}

${ }^{a}$ The superior and inferior lobes of the left lung contained an adenocarcinoma, with a previous history of gastric adenocarcinoma. The other two masses in the middle and inferior lobe of right lung were identified during a pre-operative examination for cervical cancer. ${ }^{\mathrm{b}} \mathrm{Acc}$ ( tion. MPLC, multiple primary lung cancer; p stage, pathological stage; UICC, Union for International Cancer Control. 
Table II. Immunohistochemical protein expression of the four genes in primary tumors and metastatic lymph nodes.

\begin{tabular}{|c|c|c|c|c|c|}
\hline Case no. & p16 D-value & ErbB2 D-value & p27 D-value & p53 D-value & Total D-value \\
\hline 1 & 10 & 10 & 0 & 0 & 20 \\
\hline 2 & 10 & 10 & 20 & 20 & 60 \\
\hline 3 & 0 & 20 & 10 & 10 & 40 \\
\hline 4 & 20 & 10 & 10 & 0 & 40 \\
\hline 5 & 40 & 10 & 20 & 20 & 90 \\
\hline 6 & 10 & 0 & 0 & 30 & 40 \\
\hline 7 & 30 & 0 & 0 & 10 & 40 \\
\hline 8 & 0 & 0 & 40 & 40 & 80 \\
\hline 9 & 40 & 0 & 10 & 0 & 50 \\
\hline 10 & 10 & 0 & 0 & 0 & 10 \\
\hline 11 & 10 & 0 & 10 & 40 & 60 \\
\hline 12 & 0 & 10 & 10 & 0 & 20 \\
\hline 13 & 20 & 10 & 0 & 20 & 30 \\
\hline 14 & 30 & 10 & 20 & 0 & 60 \\
\hline 15 & 40 & 0 & 30 & 10 & 80 \\
\hline 16 & 20 & 0 & 0 & 40 & 60 \\
\hline 17 & 10 & 0 & 0 & 0 & 10 \\
\hline 18 & 20 & 0 & 0 & 0 & 20 \\
\hline 19 & 20 & 10 & 20 & 20 & 70 \\
\hline 20 & 0 & 0 & 10 & 0 & 10 \\
\hline 21 & 20 & 20 & 0 & 0 & 40 \\
\hline 22 & 0 & 0 & 30 & 10 & 40 \\
\hline 23 & 10 & 20 & 0 & 0 & 30 \\
\hline 24 & 10 & 0 & 10 & 0 & 20 \\
\hline 25 & 0 & 30 & 20 & 30 & 80 \\
\hline 26 & 20 & 0 & 0 & 20 & 40 \\
\hline 27 & 30 & 0 & 20 & 10 & 60 \\
\hline 28 & 10 & 10 & 30 & 30 & 80 \\
\hline 29 & 20 & 0 & 0 & 0 & 20 \\
\hline 30 & 0 & 0 & 0 & 20 & 20 \\
\hline
\end{tabular}

D-value, the sum of the differential expression ratios.

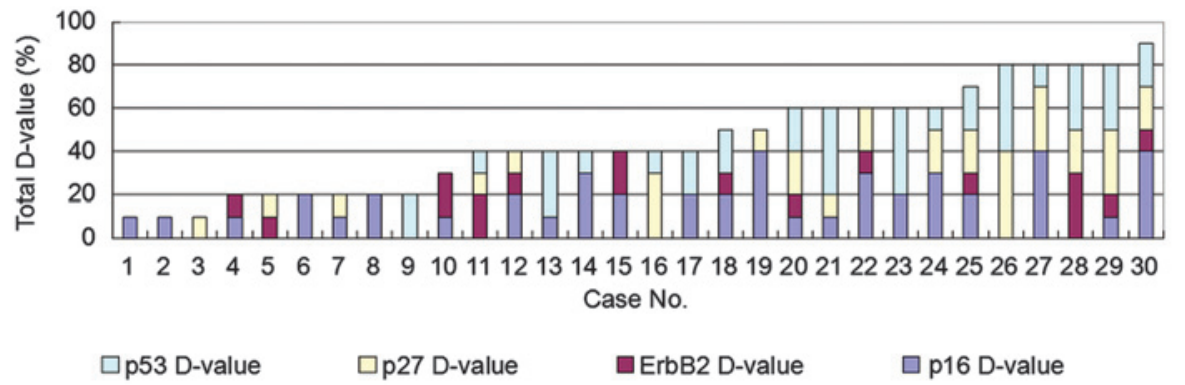

Figure 1. Immunohistochemistry was used to reveal the protein expression of four genes in the primary tumors and metastatic lymph nodes. The sum of the differential expression values (the D-value) between primary lung tumors and metastatic lymph nodes was $<90$.

metastasis, metastatic cancers or the second separate cancers. According to the experimental results, tumors can be re-diagnosed as metastatic when the sum of the differential expression ratios of the four proteins does not exceed the reference value of 90, and as MPLCs when the value does exceed 90 (Table III).

Resultsofdenovodiagnosisbaseduponadifferentially-expressed gene analysis mathematical model in MPLCs of the same histological type. Of the 36 patients with the same histological type of MPLC, who were clinically diagnosed according to the Martini and Melamed criteria (3), the sum value of the differential expression ratio was $>90$ in 29 patients $(80.5 \%)$, and $<90$ in seven patients (19.5\%) (Figs. 4 and 6). According to the model, 29 of the 36 patients $(82.0 \%)$ were diagnosed de novo with newly-classified MPLCs and seven with newly-classified IPM.

Results of de novo diagnosis based upon a differentially-expressed gene analysis mathematical model in lung 
Table III. Difference between the Martini and Melamed criteria and the mathematical model, which is based upon differentially-expressed gene analysis, in MPLC.

\begin{tabular}{lrrr}
\hline & & \multicolumn{2}{c}{ Clinical diagnosis (Martini and Melamed criteria) } \\
\cline { 2 - 4 } Differentially-expressed gene analysis & MPLC & IPM & Total \\
\hline MPLC & 29 & 6 & 35 \\
IPM & 7 & 20 & 21 \\
Total & 36 & 56 \\
\hline
\end{tabular}

Martini and Melamed criteria (3); MPLC, multiple primary lung cancer; IPM, intrapulmonary metastasis.

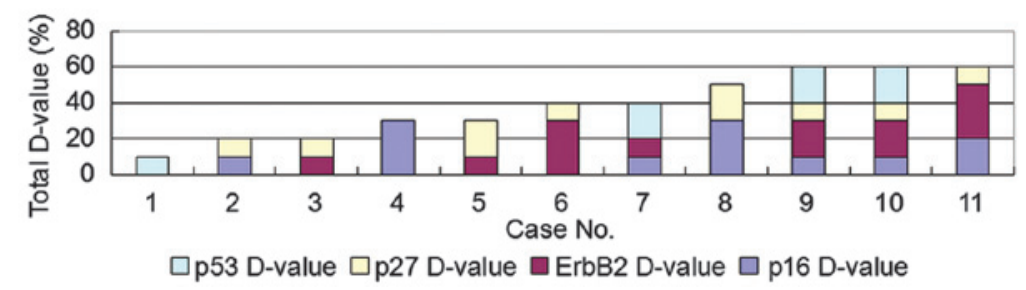

Figure 2. Immunohistochemistry was used to reveal the protein expression of four genes in the primary tumors and single metastatic foci. The sum of the differential expression values (the D-value) between the primary lung tumors and single metastatic foci was $<90$.

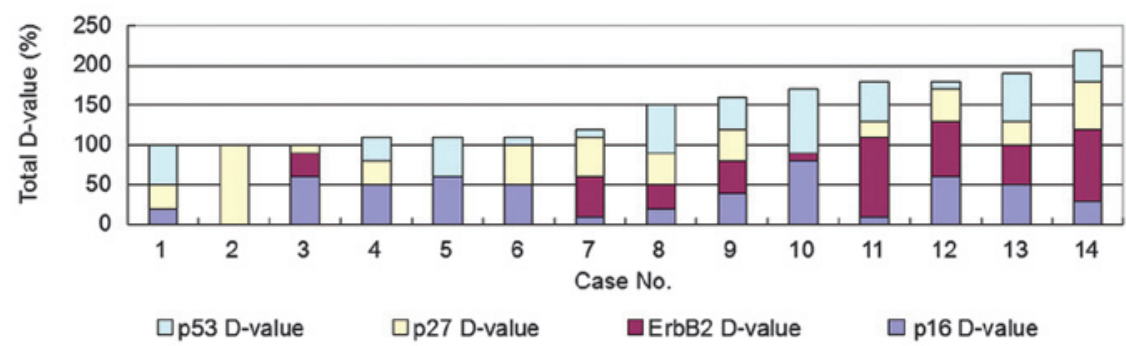

Figure 3. Immunohistochemistry was used to reveal the protein expression of four genes in the primary tumors and in the second tumor in cases of multiple primary lung cancers of different histological types. The sum of the differential expression values (the D-value) between the primary tumors and the second tumor in cases of multiple primary lung cancers of different histological types was $>90$.

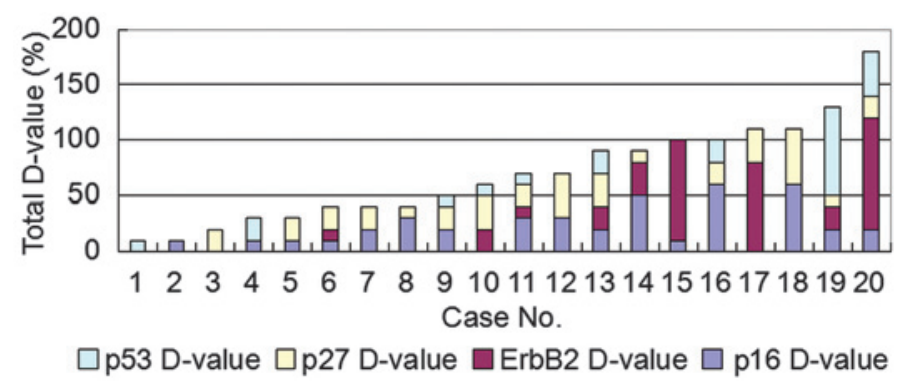

Figure 4. Immunohistochemistry was used to reveal the protein expression of four genes in the primary tumors and in the second tumor in cases of multiple primary lung cancer (MPLC) with the same histological type. According to the new classification, case nos. 1 to 14 were newly-classified as intrapulmonary metastases and case nos. 15 to 20 were newly-classified as MPLCs. The sum of the differential expression values (the D-value) between the primary tumors and the second tumor in cases of MPLC with the same histological type was $<90$ for cases 1-14 and $>90$ for cases 15-20.

cancers with IPM. Of the 20 patients with IPM who were clinically diagnosed according to the Martini and Melamed criteria (3), 14 (70.0\%) had a sum value of $\leq 90$ for the expression ratios of the four proteins. According to the same criterion, 14 of the 20 patients were diagnosed de novo with newly-classified IPM, and six with newly-classified MPLCs (Figs. 5 and 6). In total, three of the six patients $(50 \%)$ with IPM demonstrated unilateral lung lobe lesions, and the other three patients presented with bilateral lung lobe lesions. The pathological stage was diagnosed de novo as being between $\mathrm{T} 4$ and $\mathrm{T} 1$ among three of the six patients, and as M1 to T2 in the rest.

Differences in the diagnostic consistency of the mathematical model, based on differentially-expressed gene analysis and 


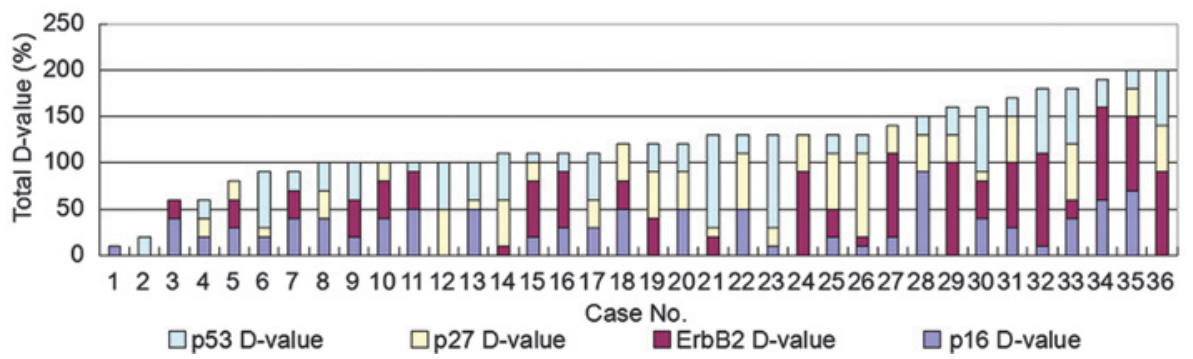

Figure 5. Immunohistochemistry was used to reveal the protein expression of four genes in the primary tumors and in the second tumor in lung cancers with intrapulmonary metastasis. According to the new classification, case nos. 1 to 7 were newly-classified as intrapulmonary metastasis and case nos. 8 to 36 were newly-classified as multiple primary lung cancers.

\section{Primary tumor Metastatic tumor}

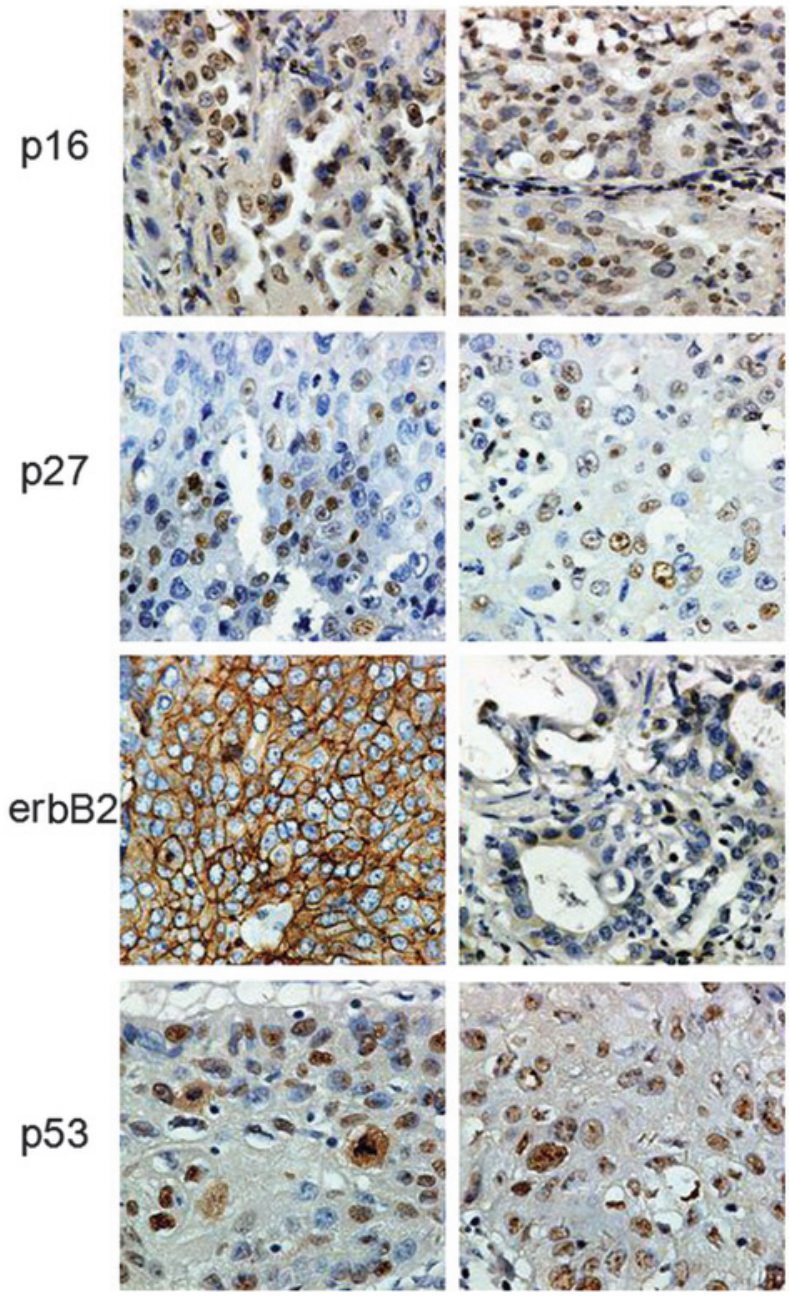

Figure 6. Immunostaining revealing the expression status of p16, p27, C-erbB2 and p53 in primary and metastatic tumors of non-small cell lung cancer. Positive expression revealed by brown-yellow nuclear staining (magnification, $\mathrm{x} 175)$.

clinical diagnosis. In total, 29 of the 36 MPLC patients were diagnosed with newly-classified MPLC and the remaining seven with newly-classified IPM. Furthermore, 14 of the 20 cases of IPM were diagnosed with newly-classified IPM and the other six with MPLC. Overall, 35 patients with multifocal lung cancer were diagnosed de novo with newly-classified MPLC, and 21 with newly-classified IPM (Table III).

\section{Discussion}

At present, individuals with lung cancer have an increased risk of developing a second lung tumor. Cases of MPLC are distinguished by the presence of a secondary neoplasm. It may be easy to diagnose cases of MPLC that exhibit different histological types. Multiple, anatomically distinct, but histologically similar lung cancers are commonly identified in the same patient. Often, it can be challenging to distinguish between cases of MPLC and IPM. The diagnostic criteria for MPLC was proposed by Martini and Melamed (3) and states that: i) MPLC tumors must occur in separate lobes or in different regions of the same lobe, ii) neoplasms may originate from different types of carcinomas in situ and demonstrate distinct histological types, and iii) no metastasis should be evident in the lymphatic system or in any other organs. However, not all patients can be classified in accordance with these guidelines. Patients with clinically diagnosed MPLCs occasionally demonstrate extremely poor five-year survival rates $(0-44 \%)$, even at stage I of the disease $(3,17-19)$. This variation in prognosis is believed to be the result of the different biological behaviors of the tumors. These results suggest that a number of patients with clinically diagnosed MPLCs may possess metastatic lesions. This indicates a potential limitation in the Martini and Melamed criteria (3), which, at present, is widely used for the clinical diagnosis of MPLCs.

A universal agreement regarding the particular approach that should be adhered to for the diagnosis of MPLC is yet to be established. Therefore, biological analyses are considered to be a useful approach for distinguishing between cases of MPLC and IPM, and for determining the correct biological stage of the lung cancer. In order to overcome this limitation, the use of clonal analyses for different tumors has been reported to discriminate between MPLCs and IPM. Previous studies have demonstrated that multiple gene analyses are able to identify the clonality in a combination of multiple gene mutations, including a p53 gene mutation, a K-ras mutation and/or LOH $(11,20-28)$. In order to differentiate between multifocal tumors and IPM, Chang et al (29) evaluated p53 somatic aberrations in MPLCs. Of the 58 patients included in the study, $22(37.9 \%)$ were identified as having the same clonality and $28(48.3 \%)$ as having different clonalities. Furthermore, it was revealed that the occurrence of lymph node metastasis was more common in lesions with the same clonality.

In the present study, IHC staining was performed in order to distinguish between MPLCs and IPM. The IHC 
expression levels of p53, p16, p27 and c-erbB2 were revealed to be significant prognostic factors for cases of lung cancer. The transcription factor, p53, is activated in response to DNA damage and is involved in cell cycle regulation, the induction of apoptosis and DNA repair. However, mutated forms of p53 are unable to effectively retain these particular functions. A mutated version or an overexpression of the p53 gene is an unfavorable prognostic factor that is observed in $~ 50 \%$ of patients with NSCLC (30). In a previous study, the presence of somatic mutations or an overexpression of $\mathrm{p} 53$ were identified in $\sim 23 \%$ and $\sim 65 \%$ of patients with NSCLC, respectively. Furthermore, p53 has been extensively investigated as a prognostic marker in cases of NSCLC, and the majority of results indicate that alterations in $\mathrm{p} 53$ are associated with a poor prognosis (31). The reproduction of human lung adenocarcinoma phenotypes in the flanks of nude mice has been successfully completed by introducing a p53 gene alternation (32). The p16 gene is also a tumor suppressor gene, which negatively regulates cell cycle progression by inhibiting cyclin-dependent kinases (CDK) 4/6. Homozygous deletions (HDs) of p16 have been frequently detected in lung cancer patients. In a previous study, HDs were detected in eight of $28(28.6 \%)$ primary tumor patients, including two of eight $(25.0 \%)$ non-invasive bronchioloalveolar carcinomas, and five of 22 (22.7\%) brain metastases (33). In another study, abnormal hypermethylation of the p16 promoter was detected in several tumors types, and was revealed to be inactivated in $40-70 \%$ of patients with NSCLC (34). The contribution of p16 deregulation via alterations in methylation during the carcinogenic process has been extensively investigated. p16 hypermethylation is considered to be an independent prognostic factor for poor patient outcomes (35). p27 is a CDK inhibitor, which is involved in the regulation of the cell cycle. By inhibiting retinoblastoma phosphorylation, $\mathrm{p} 27$ is able to suppress the progression of the cell cycle from the $\mathrm{G}_{1}$ phase to the $\mathrm{S}$ phase. A reduced expression of p27 is observed in $70-80 \%$ of patients with NSCLC. In previous studies, this particular reduced-expression group demonstrated a poorer prognosis compared with patients from a positive-expression group $(15,36,37)$. By contrast, high p27 expression is associated with an improved prognosis (38). The c-erbB-2 (HER-2/neu) proto-oncogene codes for transmembrane receptor tyrosine kinases, such as epidermal growth factor and human epidermal growth factor receptor 2 , 3 and 4, which are members of the class 1 receptor tyrosine kinase family. An overexpression of c-erbB2 is often observed in patients with NSCLC. In a previous study, c-erbB2 overexpression was identified in $37 \%$ of lung adenocarcinomas cases that were associated with a higher disease stage and a positive nodal status. Therefore, c-erbB2 has been suggested to be a potential tumor progression marker in NSCLC patients, and one that can be observed at the protein level $(39,40)$.

Two important mechanisms have been proposed, through which histologically-similar, multifocal tumors are believed to arise: i) A single clonal event occurs, which results in a tumor that subsequently spreads within one or two lungs; and ii) multiple tumors arise independently in a carcinogen-damaged field (41). The difference in protein expression between the histologically-similar tumors was hypothesized to be larger in MPLC patients, due to the various clonal origins. The D-value of protein expression in IPM patients, however, would be smaller. According to the concept of field cancerization, tissues from different fields may conduct similar or dissimilar DNA damage under the control of a carcinogen. The possibility that separate MPLC tumors may contain a similar genotype could be a default from the probability theory. When two or more separate tumors share one genotype, they are likely to be IPMs $(41,42)$.

Based on our preliminary experiments and the results of a literature review, the reliability of a single gene marker appeared to be low. Therefore, four markers, p53, p16, p27 and c-erbB2, were selected in order to distinguish between cases of MPLC and IPM, according to the early or late emergence of the gene mutation, the stability of the mutation and the correlation with prognosis. In the present study, the results indicated that when the difference between two tumors was $>90$, the patient could be newly classified as having MPLC. The 14 patients diagnosed with MPLCs of different histological types had a sum value of $>90$ for the differential expression ratios of the four proteins, which was concomitant with our hypothesis. By contrast, when the difference was $<90$, the patient was newly classified with IPM. Therefore, 36 of the MPLCs cases of the same histological type and 20 of IPM (based on the Martini and Melamed criteria) were reclassified using this novel criteria.

The results of the present study demonstrated that IHC analyses of differential protein expression profiles of multiple genes can be used to indicate the clonal origins of multiple separate tumors, and therefore facilitate the discrimination between a secondary primary cancer and IPM. As a classical pathological examination method, IHC has a number of merits, including convenience and sensitivity. For the patients diagnosed with MPLCs, particularly those with the same histological type, it was challenging to determine a correct diagnosis of MPLC or IPM based entirely on the Martini and Melamed criteria (3). Therefore, an IHC test should be performed in order to confirm the correctly diagnosed ratios. The quantitative differentially-expressed gene mathematical model is considered to be a useful approach for distinguishing between MPLCs of the same histological type and IPM. The precise discrimination between MPLC and IPM should enable rationalized treatment strategies, and improve the prognoses of the affected patients. However, as the number of analyzed cases in the present study was relatively small, future studies with larger cohorts will be required in order to confirm these results.

\section{Acknowledgements}

This study was supported by grants from the National Science Foundation (no. 81071929) and the Wu Jieping Medical Foundation (no. 320.6799.1120).

\section{References}

1. Ferguson MK, DeMeester TR, DesLauriers J, et al: Diagnosis and management of synchronous lung cancers. J Thorac Cardiovasc Surg 89: 378-385, 1985.

2. Adebonojo SA, Moritz DM and Danby CA: The results of modern surgical therapy for multiple primary lung cancers. Chest 112: 693-701, 1997.

3. Martini $\mathrm{N}$ and Melamed MR: Multiple primary lung cancers. J Thorac Cardiovasc Surg 70: 606-612, 1975.

4. Sugio K, Kishimoto Y, Virmani AK, et al: K-ras mutations are a relatively late event in the pathogenesis of lung carcinomas. Cancer Res 54: 5811-5815, 1994. 
5. Kishimoto Y, Sugio K, Hung JY, et al: Allele-specific loss in chromosome $9 \mathrm{p}$ loci in preneoplastic lesions accompanying non-small-cell lung cancers. J Natl Cancer Inst 87: 1224-1229, 1995.

6. Wistuba II, Behrens C, Milchgrub S, Bryant D, Hung J, Minna JD and Gazdar AF: Sequential molecular abnormalities are involved in the multistage development of squamous cell lung carcinoma. Oncogene 18: 643-650, 1999.

7. Aoyagi Y, Yokose T, Minami Y, et al: Accumulation of losses of heterozygosity and multistep carcinogenesis in pulmonary adenocarcinoma. Cancer Res 61: 7950-7954, 2001.

8. Strong MS, Incze J and Vaughan CW: Field cancerization in the aerodigestive tract - its etiology, manifestation, and significance. J Otolaryngol 13: 1-6, 1984.

9. Matsuzoe D, Hideshima T, Ohshima K, et al: Discrimination of double primary lung cancer from intrapulmonary metastasis by p53 gene mutation. Br J Cancer 79: 1549-1552, 1999.

10. Hiroshima K, Toyozaki T, Kohno H, Ohwada H and Fujisawa T: Synchronous and metachronous lung carcinomas: molecular evidence for multicentricity. Pathol Int 48: 869-876, 1998

11. Mitsudomi T, Yatabe Y, Koshikawa T, et al: Mutations of the P53 tumor suppressor gene as clonal marker for multiple primary lung cancers. J Thorac Cardiovasc Surg 114: 354-360, 1997.

12. Mountain CF: Revisions in the International System for Staging Lung Cancer. Chest 111: 1710-1717, 1997.

13. Tsukamoto S, Sugio K, Sakada T, et al: Reduced expression of cell-cycle regulator p27 (Kip1) correlates with a shortened survival in non-small cell lung cancer. Lung Cancer 34: 83-90, 2001.

14. Osaki T, Mitsudomi T, Oyama T, Nakanishi R and Yasumoto K: Serum level and tissue expression of c-erbB-2 protein in lung adenocarcinoma. Chest 108: 157-162, 1995.

15. Dobashi K, Sugio K, Osaki T, Oka T and Yasumoto K Micrometastatic P53-positive cells in the lymph nodes of non-small-cell lung cancer: prognostic significance. J Thorac Cardiovasc Surg. 114: 339-346, 1997.

16. Taga S, Osaki T, Ohgami A, et al: Prognostic value of the immunohistochemical detection of p16INK4 expression in non small cell lung carcinoma. Cancer 80: 389-395, 1997.

17. Pommier RF, Vetto JT, Lee JT and Johnston KM: Synchronous non-small cell lung cancers. Am J Surg 171: 521-524, 1996.

18. Mathisen DJ, Jensik RJ, Faber LP and Kittle CF: Survival following resection for second and third primary lung cancers. J Thorac Cardiovasc Surg 88: 502-510, 1984.

19. Rosengart TK, Martini N, Ghosn P and Burt M: Multiple primary lung carcinomas: prognosis and treatment. Ann Thorac Surg 52: 773-779, 1991

20. Lau DH, Yang B, Hu R and Benfield JR: Clonal origin of multiple lung cancers: K-ras and p53 mutations determined by nonradioisotopic single-strand conformation polymorphism analysis. Diagn Mol Pathol. 6: 179-184, 1997.

21. Wang X, Christiani DC, Mark EJ, et al: Carcinogen exposure, p53 alteration, and K-ras mutation in synchronous multipleprimary lung carcinoma. Cancer 85: 1734-1739, 1999.

22. Ribeiro U, Safatle-Ribeiro AV, Posner MC, et al: Comparative p53 mutational analysis of multiple primary cancers of the upper aerodigestive tract. Surgery 120: 45-53, 1996.

23. Leong PP, Rezai B, Koch WM, et al: Distinguishing second primary tumors from lung metastases in patients with head and neck squamous cell carcinoma. J Natl Cancer Inst 90: 972-977, 1998.

24. Califano J, Leong PL, Koch WM, et al: Second esophageal tumors in patients with head and neck squamous cell carcinoma: an assessment of clonal relationships. Clin Cancer Res 5: $1862-1867,1999$
25. van Oijen MG, Leppers Vd Straat FG, Tilanus MG and Slootweg PJ: The origins of multiple squamous cell carcinoma in the aerodigestive tract. Cancer 88: 884-893, 2000.

26. Shimizu S, Yatabe Y, Koshikawa T, et al: High frequency of clonally related tumors in cases of multiple synchronous lung cancers as revealed by molecular diagnosis. Clin Cancer Res 6 : 3994-3999, 2000.

27. Sozzi G, Miozzo M, Pastorino U, et al: Genetic evidence for an independent origin of multiple preneoplastic and neoplastic lung lesions. Cancer Res 55: 135-140, 1995.

28. van der Sijp JR, van Meerbeeck JP, Maat AP, et al: Determination of the molecular relationship between multiple tumors within one patient is of clinical importance. J Clin Oncol 20: 1105-1114, 2002.

29. Chang YL, Wu CT, Lin SC, Hsiao CF, Jou YS and Lee YC: Clonality and prognostic implications of p53 and epidermal growth factor receptor somatic aberrations in multiple primary lung cancers. Clin Cancer Res 13: 52-58, 2007.

30. Mitsudomi T, Hamajima N, Ogawa $\mathrm{M}$ and Takahashi T: Prognostic significance of p53 alterations in patients with non-small cell lung cancer: a meta-analysis. Clin Cancer Res 6: 4055-4063, 2000.

31. Mogi A and Kuwano H: TP53 mutations in nonsmall cell lung cancer. J Biomed Biotechnol 2011: 583929, 2011.

32. Sasai K, Sukezane T, Yanagita E, et al: Oncogene-mediated human lung epithelial cell transformation produces adenocarcinoma phenotypes in vivo. Cancer Res 71: 2541-2549, 2011.

33. Iwakawa R, Kohno T, Anami Y, et al: Association of p16 homozygous deletions with clinicopathologic characteristics and EGFR/KRAS/p53 mutations in lung adenocarcinoma. Clin Cancer Res 14: 3746-3753, 2008.

34. Otterson GA, Kratzke RA, Coxon A, Kim YW and Kaye FJ: Absence of p16INK4 protein is restricted to the subset of lung cancer lines that retains wildtype RB. Oncogene 9: 3375-3378, 1994.

35. Lou-Qian Z, Rong Y, Ming L, et al: The prognostic value of epigenetic silencing of p16 gene in NSCLC patients: a systematic review and meta-analysis. PLoS One 8: e54970, 2013.

36. Esposito V, Baldi A, De Luca A, et al: Prognostic role of the cyclin-dependent kinase inhibitor p27 in non-small cell lung cancer. Cancer Res 57: 3381-3385, 1997.

37. Catzavelos C, Tsao MS, DeBoer G, et al: Reduced expression of the cell cycle inhibitor p27Kip1 in non-small cell lung carcinoma: a prognostic factor independent of Ras. Cancer Res 59: 684-688, 1999 .

38. Zhuang Y, Yin HT, Yin XL, Wang J and Zhang DP: High p27 expression is associated with a better prognosis in East Asian non-small cell lung cancer patients. Clin Chim Acta 412: 2228-2231, 2011

39. Kristiansen G, Yu Y, Petersen S, et al: Overexpression of c-erbB2 protein correlates with disease-stage and chromosomal gain at the c-erbB2 locus in non-small cell lung cancer. Eur J Cancer 37: 1089-1095, 2001.

40. Turken O, Kunter E, Cermik H, Isitmangil T, Kandemir G, Yaylaci $\mathrm{M}$ and Ozturk A: Prevalence and prognostic value of c-erbB2 expression in non-small cell lung cancer (NSCLC). Neoplasma 50: 257-261, 2003.

41. Gazdar AF and Minna JD: Multifocal lung cancers - clonality vs field cancerization and does it matter? J Natl Cancer Inst 101: $541-543,2009$

42. Dali C, Huan X and Guowei C: Differential diagnosis of multiple tumor: multiple primary cancer or metastatic cancer of lung. Chin J Cancer Prev Treat 9: 721-724, 2014. 